bioRxiv preprint doi: https://doi.org/10.1101/2020.05.27.120402; this version posted May 30, 2020. The copyright holder for this preprint (which was not certified by peer review) is the author/funder. All rights reserved. No reuse allowed without permission.

\title{
Genomic analysis of early SARS-CoV-2 strains introduced in Mexico
}

Blanca Taboada ${ }^{1^{*}}$, Joel Armando Vazquez-Perez ${ }^{2^{*}}$, José Esteban Muñoz Medina ${ }^{3^{*}}$, Pilar Ramos Cervantes $^{4^{*}}$, Marina Escalera-Zamudio ${ }^{5^{*}}$, Celia Boukadida ${ }^{6}$, Alejandro Sanchez-Flores ${ }^{7}$, Pavel Isa ${ }^{1}$, Edgar Mendieta Condado ${ }^{8}$, José Arturo Martínez-Orozco ${ }^{2}$, Eduardo Becerril-Vargas ${ }^{2}$, Jorge Salas-Hernández ${ }^{2}$, Ricardo Grande ${ }^{7}$, Carolina González-Torres ${ }^{9}$, Francisco Javier Gaytán-Cervantes ${ }^{9}$, Gloria Vazquez, Francisco Pulido ${ }^{7}$, Adnan Araiza Rodríguez ${ }^{8}$, Fabiola Garcés Ayala ${ }^{8}$, Cesar Raúl González Bonilla ${ }^{10^{\prime}}$, Concepción Grajales Muñiz ${ }^{11}$, Víctor Hugo Borja Aburto ${ }^{12}$, Gisela Barrera Badillo ${ }^{8}$, Susana López ${ }^{1}$, Lucía Hernández Rivas ${ }^{8}$, Rogelio Perez-Padilla ${ }^{2}$, Irma López Martínez ${ }^{8}$, Santiago Ávila-Ríos ${ }^{6}$, Guillermo RuizPalacios ${ }^{4}$, José Ernesto Ramírez-González ${ }^{8+}$, and Carlos F. Arias ${ }^{1+}$

${ }^{1}$ Departamento de Genética del Desarrollo y Fisiología Molecular, Instituto de Biotecnología, Universidad Nacional Autónoma de México, Cuernavaca, Morelos, Mexico; ${ }^{2}$ Instituto Nacional de Enfermedades Respiratorias Ismael Cosío Villegas, Ciudad de México, Mexico; ${ }^{3}$ División de Laboratorios de Vigilancia e Investigación Epidemiológica, Instituto Mexicano del Seguro Social, Ciudad de México, México; ${ }^{4}$ Instituto Nacional de Ciencias Médicas y Nutrición, Ciudad de México, Mexico; ${ }^{5}$ Department of Zoology, Oxford University, Oxford, UK; ${ }^{6}$ Centro de Investigación en Enfermedades Infecciosas, Instituto Nacional de Enfermedades Respiratorias Ismael Cosío Villegas, Ciudad de México, Mexico; ${ }^{7}$ Unidad Universitaria de Secuenciación Masiva y Bioinformática, Instituto de Biotecnología, Universidad Nacional Autónoma de México, Cuernavaca, Morelos, Mexico; ${ }^{8}$ Instituto de Diagnóstico y Referencia Epidemiológicos, Dirección General de Epidemiología, Ciudad de México, Mexico; ' División de Desarrollo de la Investigación, Instituto Mexicano del Seguro Social, Ciudad de México, México; ${ }^{10}$ Coordinación de Investigación en Salud, Instituto Mexicano del Seguro Social, Ciudad de México, México; ${ }^{11}$ Coordinación de Control Técnico de Insumos, Instituto Mexicano del Seguro Social, Ciudad de México, México; ${ }^{12}$ Dirección de Prestaciones Médicas, Instituto Mexicano del Seguro Social, Ciudad de México, Mexico.

*Blanca Taboada, Joel Armando Vazquez-Perez, José Esteban Muñoz Medina, Pilar Ramos Cervantes, and Marina Escalera-Zamudio contributed equally to this work

This work is the result of the collaboration of five institutions into one research consortium: three public health institutes and two universities. From the beginning of this work, it was agreed that the experimental leader of each institution would share the first authorship. Those were the criteria followed to assign first co-first authorship in this manuscript. The order of the other authors was randomly assigned.

+Corresponding authors: José Ernesto Ramírez-González, and Carlos F. Arias

JERG: Instituto de Diagnóstico y Referencia Epidemiológicos, Dirección General de Epidemiología, Ciudad de México, Mexico. e-mail: ernesto.ramirez@salud.gob.mx

CFA: Departamento de Genética del Desarrollo y Fisiología Molecular, Instituto de Biotecnología, Universidad Nacional Autónoma de México, Cuernavaca, Morelos, Mexico. e-mail: arias@ibt.unam.mx

Running Titer: SARS-CoV-2 genome sequences in Mexico 


\section{ABSTRACT}

50 The COVID-19 pandemic has affected most countries in the world. Studying the evolution and transmission patterns in different countries is crucial to implement effective strategies for disease control and prevention. In this work, we present the full genome sequence for 17 SARS-CoV-2 isolates corresponding to the earliest sampled cases in Mexico. Global and local phylogenomics, coupled with mutational analysis, consistently revealed that these viral sequences are distributed within 2 known lineages, the SARS-CoV-2 lineage A/G, containing mostly sequences from North America, and the lineage B/S containing mainly sequences from Europe. Based on the exposure history of the cases and on the phylogenomic analysis, we characterized fourteen independent introduction events. Additionally, three cases with no travel history were identified. We found evidence that two of these cases represent local transmission cases occurring in Mexico during mid-March 2020, denoting the earliest events described in the country. Within this Mexican cluster, we also identified an H49Y amino acid change in the spike protein. This mutation is a homoplasy occurring independently through time and space, and may function as a molecular marker to follow on any further spread of these viral variants throughout the country. Our results depict the general picture of the SARS-CoV-2 variants introduced at the beginning of the outbreak in Mexico, setting the foundation for future surveillance efforts.

\section{IMPORTANCE}

Understanding the introduction, spread and establishment of SARS-CoV-2 within distinct human populations is crucial to implement effective control strategies as well as the evolution of the pandemics. In this work, we describe that the initial virus strains introduced in Mexico came from Europe and the United States and the virus was circulating locally in the country as early as mid-March. We also found evidence for early local transmission of strains having the mutation H49Y in the Spike protein, that could be further used as a molecular marker to follow viral spread within the country and the region. 


\section{INTRODUCTION}

75

The 2019 coronavirus disease (COVID-19), declared a pandemic by the WHO on March $11^{\text {th }}, 2020^{1}$, is caused by a novel betacoronavirus known as the severe acute respiratory syndrome coronavirus 2 (SARS-CoV-2), detected in December of 2019 in the province of Wuhan in China ${ }^{1}$. This is the third outbreak related to zoonotic betacoronaviruses known to occur in humans in the last two decades, after SARS (severe acute respiratory syndrome) in 2002 and MERS (Middle East respiratory syndrome) in 2012. After its emergence in China, SARS-CoV-2 spread initially to other parts of the world by people with a travel history to China, but gradually shifted to local transmissions ${ }^{2}$. Viral spread was first detected in Thailand, South Korea and Japan, and by the second half of January, the first positive cases appeared in the USA and Europe (France, Italy and Spain). The current SARS-CoV-2 genome analysis in the Nextstrain site ${ }^{3}$, points out that viral transmission is now mainly community-driven ${ }^{4,5}$.

In many countries, despite diagnostic efforts and initial control strategies, SARS-CoV-2 spread went on undetected until a critical number of cases requiring hospitalization and intensive care was reached, alerting the authorities in charge. As for May $4^{\text {th }} 2020$, SARS-CoV-2 has infected more than 3,578,000 people and caused around 251,000 deaths worldwide ${ }^{3}$. In Mexico, the first case of SARS-CoV2 was detected on February $27^{\text {th }} 2020$, corresponding to a person who travelled back to Mexico from Italy, and who was in direct contact with a confirmed SARS-CoV-2 case. Soon after, additional cases were detected among travelers that returned from the USA and Europe, increasing every day. By May $4^{\text {th }}$, there were over 23,400 confirmed cases and 2,150 deaths within the country, indicating local transmission ${ }^{6}$. Understanding the introduction, spread and establishment of SARS-CoV-2 within distinct human populations is crucial to implement effective control strategies. In this work, we studied the early introduction dynamics of the first SARS-CoV-2 cases in Mexico. For this, we used a whole genome sequencing and phylogenomic approach. We obtained 17 full viral genome sequences, including the first case detected and sampled within the country. Phylogenomic placement showed that these viruses belong to the $A 2 / G$ and $B / S$ lineages, two of the three circulating viral lineages reported so far. Our analysis also confirms that there have been multiple independent introduction events in Mexico from travelers abroad. We also found evidence for early local transmission of strains having the mutation H49Y in the Spike protein, that could be further used as a molecular marker to follow viral spread within the country. 
All clinical samples were processed at the "Instituto de Diagnóstico y Referencia Epidemiológicos" (InDRE), following official procedures ${ }^{7}$. All samples used for this work are considered part of the national response to COVID-19, and the data collected is directly related to disease control.

\section{Sample collection and diagnostics}

111 All samples used in this study were collected under the Mexican Official Norm NOM-024-SSA2-1994 for

112 prevention and control of acute respiratory infections in the primary health attention, as part of the early diagnostics scheme for SARS-CoV-2 in public health laboratories and hospitals in Mexico City (Red

114 Nacional de Laboratorios Estatales de Salud Pública, RNLSP; Instituto Nacional de Enfermedades 115 Respiratorias, INER; Instituto Nacional de Ciencias Médicas y Nutrición Salvador Zubirán, INCMNSZ; and 116 Instituto Mexicano del Seguro Social, IMSS). Oro- and naso-pharyngeal swabs were collected and placed 117 in virus transport medium upon collection, following InDRE official procedures ${ }^{8}$. A tracheal aspirate was 118 also obtained from one patient and it was frozen at $-70^{\circ} \mathrm{C}$ until use. Diagnosis was done using validated protocols for SARS-CoV 2, as approved by InDRE and by the World Health Organization (WHO) ${ }^{9}$.

\section{Sample processing and Whole Genome Sequencing}

122 All samples were prepared for RNA extraction, as described ${ }^{10,11}$. Briefly, centrifuged and filtered supernatants were treated with Turbo DNase and RNAse. Nucleic acids were then extracted using the

124 PureLink ${ }^{\mathrm{TM}}$ Viral RNA/DNA Kit (ThermoFisher), following the manufacturer's instructions and using linear acrylamide (Ambion) as RNA carrier. cDNA was synthesized using the SuperScript III Reverse

126 Transcriptase System (ThermoFisher) and primer A (5'-GTTTCCCAGTAGGTCTCN9-3'). The second strand was generated by two rounds of synthesis with Sequenase 2.0, followed by 15 cycles of amplification using Phusion DNA polymerase using primer B (5'-GTTTCCCAGTAGGTCTC-3). Next, cDNA was purified using the DNA Clean \& Concentrator Kit (Zymo Research) and used as input material

130 for generating sequencing libraries, following the Nextera XT DNA Library Preparation Kit ${ }^{12}$ (Illumina) .

131 Finally, all samples were sequenced on the Illumina NextSeq 500 platform using a 150 cycle High Output

132 Kit v2.5 to obtain paired end reads of 75 base pairs. Sequencing yields are reported in SI Table 1. 


\section{Bioinformatic analysis}

135 Data quality control and processing

136 Read quality control was carried out using FAST-QC ${ }^{13}$ under the default parameters. Adapter sequences 137 and low-quality bases were removed using Fastp v0.19 $9^{14}$. Low complexity reads, those with a length 138 shorter than 40 bases, and duplicates were excluded using CD-HIT-DUP v.4.6. $8^{15}$. Off-target reads were 139 then filtered out using Bowtie2 v2.3.4.3 with the default parameters against the human genome version 140 GRCh38.p13, and the SILVA database ${ }^{16}$ as a reference to filter out human DNA and ribosomal 141 sequences.

Viral genome assembly

144 The reads obtained were used as input to assemble viral genomes using the Wuhan-Hu-1 reference genome sequence (MN908947). For this, the reads obtained for each sample were mapped against the reference using Bowtie2 v2.3.4.3. Aligned reads were then used for de novo assembly using SPAdes $v^{17}$.

Consensus genome sequences were generated using the majority threshold criterion. Only sequences with a coverage above $80 \%$ and a mean depth of $\geq 8 \mathrm{X}$ were considered for the analyses (SI Table 1 ).

\section{Phylogenetic analyses}

151 Data collation

152 From 4698 complete SARS-CoV-2 genomes deposited in the Global Initiative on Sharing All Influenza

153 Data (GISAID) platform on the morning of April $7^{\text {th }} 2020$, a total of 3014 sequences genomes $(>29,000 \mathrm{nt}$ and only high coverage) were downloaded to generate a local database. As the collection dates of the Mexican samples range from late February to March, we filtered sequences collected between $02 / 01 / 2020$ to $03 / 31 / 2020$ for our database (Table 1). From these, unique sequences were extracted and those identical were collapsed, leaving 2633. We then included the 17 consensus viral genomes determined in this study and the Wuhan-Hu-1 reference genome sequence, yielding a total of 2651 sequences. We aligned the whole genome nt dataset using MUSCLE v3. $8^{18}$ under default parameters, and then used the getorfs script from the EMBOSS suite ${ }^{19}$ to extract complete ORFs (Open Reading

161 Frames) above 300 nt (Orf1a, Orf1b, Spike, M, Orf3a, Orf7a, Orf8 and N), that were then individually realigned as described ${ }^{18}$. Finally, to exclude UTRs and non-coding intergenic regions from the 
163 phylogenomic analyses, individual ORFs were concatenated to generate an additional $28,320 \mathrm{nt}$-long whole genome (WG) alignment.

165

Data subsampling and tree inference

167 The individual and concatenated alignments were then reversed to nucleotides and used for estimating maximum likelihood (ML) trees using RAxML v8 ${ }^{20}$ under the following parameters: $-T 2$-f a -x $390-m$ GTRGAMMA -p 580 -N 100. All trees were rooted on Wuhan-Hu-1 reference genome sequence ${ }^{21}$. Given that the SARS-CoV-2 virus shows a low degree of genetic variation ${ }^{22}$, clade definition must be based on consensus branching patterns within different trees and by shared nucleotide substitution patterns ${ }^{23}$, in addition to bootstrap support values. Based on these criteria, the position of the Mexican sequences was determined within the whole genome (WG) and individual ORF1a, ORF1b and S trees (SI Table 2), and was then confirmed on the global phylogeny available in Nextstrain ${ }^{24}$.

To visualize details on the phylogenetic relatedness of the Mexican sequences, we then subsampled the previous large-scale WG alignment in a phylogenetically-informed manner, as based on

177 the position of the Mexican strains within the large-scale trees and by selecting sequences using pairwise 178 genetic distances ${ }^{25,26}$. Briefly, all Mexican sequences were retained together with their immediate 179 ancestors and descendants, and 184 sequences were further selected based on the minor pairwise genetic distance in relation to the Mexican genomes under a threshold of $99.5 \%$ (SI Table 3). The subsampled WG alignment was scanned for recombinant sequences using the GARD algorithm ${ }^{27}$ in the Datamonkey server ${ }^{28,29}$. A total of 201 sequences, including the Wuhan-Hu-1 reference genome, were used to re-estimate subsampled trees, as described above.

Global phylogenetic analysis and shared nucleotide substitution patterns ${ }^{23}$ were used to confirm the position of the Mexican sequences based on the consensus clustering patterns observed within the large-scale WG tree, the subsampled WG tree, and the individual large-scale ORF trees, using a

187 bootstrap value $>50$ for branch support, when possible (SI Table 2). In general, we observed consistency within the global tree and our large-scale and subsampled trees, as depicted by a conserved general structure at an internal branch level. Finally, analysis of the phylogenetic relationship between the Mexican and other viral sequences to identify groups of introduction events (IE) and local transmissions (LT) was done based on the following local definition, in which and IE or LT must include: i) one or more 
192 Mexican sequences, ii) a minimum of one closest related sister sequence(s), iii) and/or the immediate common ancestor ${ }^{30,31}$.

Mutation identification

196 Snippy $^{32}$ was used to identify all mutations unique to the Mexican genomes, as compared to the reference genome sequence of isolate Wuhan-Hu-1. The large-scale WG alignment in nucleotides (including UTR and intergenic regions) was used as input for SI Table 4, while the large-scale WG concatenated ORF alignment was used as input for SI Table 5. The frequency and distribution for nucleotide and amino acid changes were determined using a normalized Sequence Logo, implemented by JalView ${ }^{33}$. Non-conservative amino acid changes were determined based on amino acid properties. Finally, the list of amino acid changes obtained was compared to the list of known sites scored to be evolving under pervasive, episodic or directional positive selection, as tested under several $d N / d S$ models $^{34,35}$.

205

RESULTS AND DISCUSSION

207

\section{Multiple introduction events of SARS-CoV-2 variants from two different lineages}

A total of 17 full viral genome sequences were obtained from selected Mexican samples representing the earliest sampled cases detected in the country (Figure 1). From the epidemiological data associated with the Mexican samples, 15 of the cases corresponded to introduction events from travelers returning from abroad that entered the country through Mexico City airport, with 5 of them then relocating to other places within the country using local transportation (either aerial or terrestrial). Two additional cases reported no

214 travel history (Table 1). Global phylogenetic analysis ${ }^{23}$ confirmed that 8 Mexican strains (samples 8, 17, $19,24,27,28,30,31$ ) grouped within the SARS-CoV-2 lineage B (also called lineage S, composed by sequences predominantly from the Americas). The remaining 9 Mexican strains (samples 2, 5, 6, 7, 13, 16, 22, 32, and 33) grouped within lineage A2 and subclade A2a (also called lineage G, composed by sequences predominantly from Europe) (Figure 2) ${ }^{36}$. Both the $A 2 / G$ and $B / S$ lineages are contemporary, as the estimated date of emergence for lineage B/S is 12/29/2019 ( $\mathrm{Cl}: 12 / 20 / 2019,01 / 03 / 2020$ ), while for lineage $A 2 / G$ is $01 / 18 / 2020(\mathrm{Cl} 01 / 02 / 2020,01 / 19 / 2020)^{23}$. The collection dates for the Mexican samples that fall within lineage $B 1$ range from $03 / 04 / 2020$ to $03 / 15 / 2020$, while those that fall within lineage $A 2$ 
range from $02 / 27 / 2020$ to $03 / 15 / 2020$, including the strain corresponding to the first reported case in México $^{37}$ REF (sample 33). This suggests an initial co-circulation of both the $A 2 / G$ and $B / S$ lineages in Mexico (Figure 2). Viruses belonging to the third lineage reported, $\mathrm{V}$, were not identified in this study. phylogenetic relatedness of the Mexican sequences, due to low support values and inconsistent clustering patterns across trees (SI Table 2). The current resolution of phylogenomic analyses is limited by the low diversity of the SARS-CoV-2 virus. Thus, for the characterized Mexican viral strains, we could only determine with confidence the geographical origins at a regional level, rather than at country-level resolution. Altogether, these observations suggest that the virus variants identified in this study were more closely related to virus strains circulating at the time in the USA and Europe rather than in China or South East Asia.

\section{Evidence for early local transmission}

237 Sequences 27 and 31 correspond to two individuals that shared travel history to Vail, CO, USA, and that were in direct contact with case 28 in the return flight to Mexico. Nonetheless, the distribution of sequence 28 in an independent group (IE12) in relation to sequences 27 and 31 (Figure 2, SI Table 2), suggests that there were at least two different viral variants co-circulating within this specific location in the USA. On the other hand, sequences $8,27,30$ and 31 grouped together, representing a local transmission cluster (LT11) (Figure 2). This observation is supported by phylogenetic consistency within our local trees and the global tree ${ }^{23}$, and a high support value (bs: 100) observed for LT11 in all cases (S1 Table 2,

244 Figure 2). Case 8 corresponds to an individual with no epidemiological relationship or contact with cases 27, 30 and 31, and with no travel history. Case 30 had travel history to Madrid, Spain, but no direct exposure with cases 27 and 31 . For case 30 , the possibility of this person acquiring the virus whilst being abroad cannot be ruled out. However, our analysis shows evidence that this person may have contracted the virus whilst already being in Mexico. Thus, LT11 strongly supports the occurrence of at least one independent local transmission event in Mexico City (case 8), as early as the second week of March. 
252 When compared to the Wuhan-Hu-1 reference genome, the Mexican sequences displayed between 4 and 10 nucleotide substitutions, and between 1 and 5 amino acid changes. This is consistent with the reported rate of evolution of $\sim 8 \times 10^{-4}$ nucleotide substitutions per site per year, equivalent to $\sim 2$ substitutions per month ${ }^{38,39}$. Collectively, 46 nucleotide substitutions and 20 amino acid residues were identified within the Mexican viral genomes (SI Table 4 and 5). As expected, the majority of these variants were not conserved through the genomes, and only 15 nucleotide changes and 6 amino acid changes were shared by more than one sequence. These results exclude sequences 6,13 and 16 , which showed a considerably lower coverage and depth when compared to the remaining 14 high-quality viral genomes obtained. Thus, most of the variability observed could be explained by errors introduced during reverse transcription, PCR amplification, sequencing or assembly (SI Table 1 and 4). showed two clade-specific nucleotide substitutions, C241T and A23403G. A23403G results in the D614Ge amino acid change in the Spike protein (Table 2). All lineage A2a sequences (including the Mexican isolates) had the additional nucleotide substitution C14408T, resulting in the amino acid change P314L in Orf1 $\mathrm{b}^{23}$. Sequences within lineage B had the nucleotide substitution T28144C rendering the L84S amino acid change in the Orf8 protein. Similarly, lineage B1 sequences also showed the nucleotide substitution C18060T (Table 2). No evidence for recombination was found within any of the alignments, in agreement with previous observations ${ }^{28}$. Taken together, our results suggest the Mexican viral sequences display the genetic changes according to their phylogenetic placement.

271

\section{H49Y amino acid change in Mexican sequences within LT1}

273 We further identified within all Mexican sequences grouping with the local transmission cluster (LT11), an $274 \mathrm{H}$ to $\mathrm{Y}$ amino acid change in position 49 of the Spike protein, corresponding to the C21707T nucleotide change in the whole genome and nucleotide alignment numbering. H49Y represents a non-conservative mutation located within the $\mathrm{N}$-terminal domain (NTD) of the $\mathrm{S}$ glycoprotein $\operatorname{trimer}^{40}$. No evidence of episodic or directional positive selection was found for this site, as tested under several $d N / d S$ models $^{34,35}$.

Mapping this nucleotide change onto the Nextstrain global tree ${ }^{23}$ with dates ranging from

280 December 2019 to April 2020, revealed that this mutation represents a homoplasy that has occurred with a frequency of $0.4 \%(20 / 4533)$ within the genomes displayed in the global tree as of May $6^{\text {th }} 2020$. This 
282

283

284

285

286

287

288

289

290

291

292

293

294

295

296

297

298

299

300

301

302

303

304

305

306

307

308

309

310

311

change first occurred in a single cluster of 14 sequences mainly from China (representative sequence: Jiangsu/JS02/2020 EPI ISL 411952). The estimated date of emergence for this cluster that eventually died off (stopped circulating and had no more descendants) was of 01/12/2020 (Cl:01/08/202001/16/2020). This mutation was detected again in the Mexican sequences in LT11 (Table 1, Figure 2). Since then, the $\mathrm{C} 21707 \mathrm{~T} / \mathrm{H} 49 \mathrm{Y}$ mutation has also appeared independently in three strains from Australia (EPI ISL 426898, EPI_ISL_430632 and EPI_ISL_430633), two strains from the UK (EPI_ISL_433757 and EPI_ISL_432818), one strain from Taiwan (EPI_ISL_417518) and two strains from the USA (EPI_ISL_408010 and EPI_ISL_430050), with collection dates ranging from 01/29/2020 to 04/05/2020 (sequences deposited on GISAID platform by May $10^{\text {th }} 2020$ ). Of notice, all the sequences displaying the H49Y mutation belong to different viral lineages (either, A2, B1 or others), confirming non-phylogenetic correlation (e.g. founder effect), but rather an independent occurrence. It would be important to follow up the genomic surveillance of homoplasic mutations that keep emerging independently through time and space, either to determine if these are sequencing errors ${ }^{41}$, or to know if these are real homoplasies that may be associated with changes in biological properties or the virus, such as increased virulence as has been shown for other viruses ${ }^{25}$. In addition, using $\mathrm{H} 49 \mathrm{Y}$ as a molecular marker could be used to follow up any further circulation and spread of these viral variants in Mexico.

\section{Data Availability}

The generated sequences SARS-CoV-2 from Mexico can be found in GISAID and in Nextstrain ${ }^{24}$. The corresponding GISAID accession numbers are listed in Table 1.

\section{ACKNOWLEDGEMENTS}

This work was partially supported by grant "Epidemiología genómica de los virus SARS-CoV-2 circulantes en México" from the National Council for Science and Technology (CONACyT)-Mexico to CFA, and by grants from the Mexican Government (Comisión de Equidad y Género de las Legislaturas LX-LXI y Comisión de Igualdad de Género de la Legislatura LXII de la H. Cámara de Diputados de la República Mexicana) to SAR and CB. MEZ is supported by a Leverhulme Trust ECR Fellowship (ECF-2019-542). We thank the "Unidad de Secuenciación Masiva y Bioinformática" of the "Laboratorio Nacional de Apoyo Tecnológico a las Ciencias Genómicas" (CONACyT \#260481) for their support in sequencing services. We thank all the staff of the Technological Development and Molecular Research Unit, Virology 
bioRxiv preprint doi: https://doi.org/10.1101/2020.05.27.120402; this version posted May 30, 2020. The copyright holder for this preprint (which was not certified by peer review) is the author/funder. All rights reserved. No reuse allowed without permission.

312 Department, and Sample Control and Services Department at InDRE for their technical assistance. The

313 findings and conclusions in this report are only the responsibility of the authors and do not necessarily of 314 the institutions involved.

315 


\section{FIGURE LEGENDS}

\section{Figure 1. Epidemiological positioning of the SARS-CoV-2 samples from Mexico}

Epidemiological curve for the early SARS-CoV-2 epidemic in Mexico, dating from late February until early April. The rise in cumulative cases is shown in red, whilst the daily incidence is shown with orange bars. Dates of collection for the samples used in this study are indicted with the black arrows. One arrow might indicate the collection of more than two samples (dates shown in Table 1).

\section{Figure 2. Phylogenomic positioning of the SARS-CoV-2 strains from Mexico.}

RAXML tree estimated from the reduced whole genome alignment built using the concatenated main viral ORFs, and rooted using the Wuhan-Hu-01 strain. Sequences corresponding to the viral isolates from Mexico sequenced in this work are shown in red, while the region of origin for the identified closest related immediate ancestors and/or sister isolates is indicated in black. Support values for the branches of interest are indicated with numbers next to the branches. The clusters identified in this work representing introduction events (IE1-IE10 and IE12 and IE13) and the local transmission (LT11) are shown next to the strain names. 


\section{REFERENCES}

1. Zhou, P. et al. A pneumonia outbreak associated with a new coronavirus of probable bat origin. Nature 579, 270-273 (2020).

2. Pinotti, F. et al. Lessons learnt from 288 COVID-19 international cases: importations over time, effect of interventions, underdetection of imported cases. medRxiv 2020.02.24.20027326 (2020).

3. auspice. https://nextstrain.org/narratives/ncov/sit-rep/2020-01-23.

4. Li, Q. et al. Early Transmission Dynamics in Wuhan, China, of Novel Coronavirus-Infected Pneumonia. N. Engl. J. Med. 382, 1199-1207 (2020).

5. Liu, J. et al. Community Transmission of Severe Acute Respiratory Syndrome Coronavirus 2, Shenzhen, China, 2020. Emerg. Infect. Dis. 26, (2020).

6. [No title]. https://www.gob.mx/cms/uploads/attachment/file/547271/Comunicado_Tecnico_Diario_COVID19_2020.04.18.pdf.

7. de Salud, S. Sistema Nacional de Vigilancia Epidemiológica. gob.mx http://www.gob.mx/salud/acciones-y-programas/sistema-nacional-de-vigilancia-epidemiologica.

8. http://www.censida.salud.gob.mx/descargas/biblioteca/documentos/manual_para_la_toma_envio_y_ recepcion_de_muestras.pdf.

9. https://www.who.int/docs/default-source/coronaviruse/protocol-v2-1.pdf?sfvrsn=a9ef618c_2.

10. Taboada, B. et al. Is There Still Room for Novel Viral Pathogens in Pediatric Respiratory Tract Infections? PLoS One 9, e113570 (2014).

11. Taboada, B. et al. The Geographic Structure of Viruses in the Cuatro Ciénegas Basin, a Unique Oasis in Northern Mexico, Reveals a Highly Diverse Population on a Small Geographic Scale. Appl. Environ. Microbiol. 84, (2018).

12. Nextera XT DNA Sample Prep Kit Documentation. https://support.illumina.com/sequencing/sequencing_kits/nextera_xt_dna_kit/documentation.html.

13. Babraham Bioinformatics - FastQC A Quality Control tool for High Throughput Sequence Data. https://www.bioinformatics.babraham.ac.uk/projects/fastqc/.

14. Chen, S., Zhou, Y., Chen, Y. \& Gu, J. fastp: an ultra-fast all-in-one FASTQ preprocessor. Bioinformatics 34, i884-i890 (2018).

15. Fu, L., Niu, B., Zhu, Z., Wu, S. \& Li, W. CD-HIT: accelerated for clustering the next-generation 
sequencing data. Bioinformatics 28, 3150-3152 (2012).

16. Quast, C. et al. The SILVA ribosomal RNA gene database project: improved data processing and web-based tools. Nucleic Acids Res. 41, D590-6 (2013).

17. Bankevich, A. et al. SPAdes: a new genome assembly algorithm and its applications to single-cell sequencing. J. Comput. Biol. 19, 455-477 (2012).

18. Edgar, R. C. MUSCLE: a multiple sequence alignment method with reduced time and space complexity. BMC Bioinformatics 5, 1-19 (2004).

19. Rice, P., Longden, I. \& Bleasby, A. EMBOSS: The European Molecular Biology Open Software Suite. Trends Genet. 16, 276-277 (2000).

20. Stamatakis, A. RAxML version 8: a tool for phylogenetic analysis and post-analysis of large phylogenies. Bioinformatics 30, 1312-1313 (2014).

21. Wu, F. et al. A new coronavirus associated with human respiratory disease in China. Nature $\mathbf{5 7 9}$, 265-269 (2020).

22. Pond, S. Genomic diversity and divergence of SARS-CoV-2/COVID-19. Observable https://observablehq.com/@spond/current-state-of-sars-cov-2-evolution (2020).

23. https://nextstrain.org/ncov/global?dmax=2020-04-08.

24. https://nextstrain.org/ncov/global.

25. Escalera-Zamudio, M. et al. Parallel Evolution in the Emergence of Highly Pathogenic Avian Influenza A Viruses. bioRxiv 370015 (2019) doi:10.1101/370015.

26. Hassan, A. S., Pybus, O. G., Sanders, E. J., Albert, J. \& Esbjörnsson, J. Defining HIV-1 transmission clusters based on sequence data. AIDS 31, 1211-1222 (2017).

27. Kosakovsky Pond, S. L., Posada, D., Gravenor, M. B., Woelk, C. H. \& Frost, S. D. W. GARD: a genetic algorithm for recombination detection. Bioinformatics 22, 3096-3098 (2006).

28. Robertson, D. I. et al. nCoV's relationship to bat coronaviruses \& recombination signals (no snakes) no evidence the 2019-nCoV lineage is recombinant. Virological http://virological.org/t/ncovsrelationship-to-bat-coronaviruses-recombination-signals-no-snakes-no-evidence-the-2019-ncovlineage-is-recombinant/331 (2020).

29. Datamonkey Adaptive Evolution Server. http://datamonkey.org/.

30. Shiino, T. et al. Molecular Evolutionary Analysis of the Influenza A(H1N1)pdm, May-September, 2009: Temporal and Spatial Spreading Profile of the Viruses in Japan. PLoS One 5, e11057 (2010). 
31. Baillie, G. J. et al. Evolutionary Dynamics of Local Pandemic H1N1/2009 Influenza Virus Lineages Revealed by Whole-Genome Analysis. J. Virol. 86, 11-18 (2012).

32. Seemann, T. tseemann/snippy. GitHub https://github.com/tseemann/snippy.

33. Waterhouse, A. M., Procter, J. B., Martin, D. M. A., Clamp, M. \& Barton, G. J. Jalview Version 2-a multiple sequence alignment editor and analysis workbench. Bioinformatics 25, 1189-1191 (2009).

34. Pond, S. Natural selection analysis of SARS-CoV-2/COVID-19. Observable https://observablehq.com/@spond/natural-selection-analysis-of-sars-cov-2-covid-19 (2020).

35. Dashboard | SARS-CoV-2 Admin. http://covid19.datamonkey.org/2020/04/01/covid19-analysis.

36. Rambaut, A. et al. A dynamic nomenclature proposal for SARS-CoV-2 to assist genomic epidemiology. bioRxiv 2020.04.17.046086 (2020) doi:10.1101/2020.04.17.046086.

37. Garcés-Ayala, F. et al. Full Genome Sequence of the first SARS-CoV-2 detected in Mexico. Arch. Virol. (2020). In press.

38. Bedford, T. et al. Cryptic transmission of SARS-CoV-2 in Washington State. medRxiv 2020.04.02.20051417 (2020).

39. Rambaut, A. et al. Phylodynamic Analysis | 176 genomes | 6 Mar 2020. Virological http://virological.org/t/phylodynamic-analysis-176-genomes-6-mar-2020/356 (2020).

40. Gui, M. et al. Cryo-electron microscopy structures of the SARS-CoV spike glycoprotein reveal a prerequisite conformational state for receptor binding. Cell Res. 27, 119-129 (2016).

41. http://virological.org/t/issues-with-sars-cov-2-sequencing-data/473. 


\section{Figure 1}

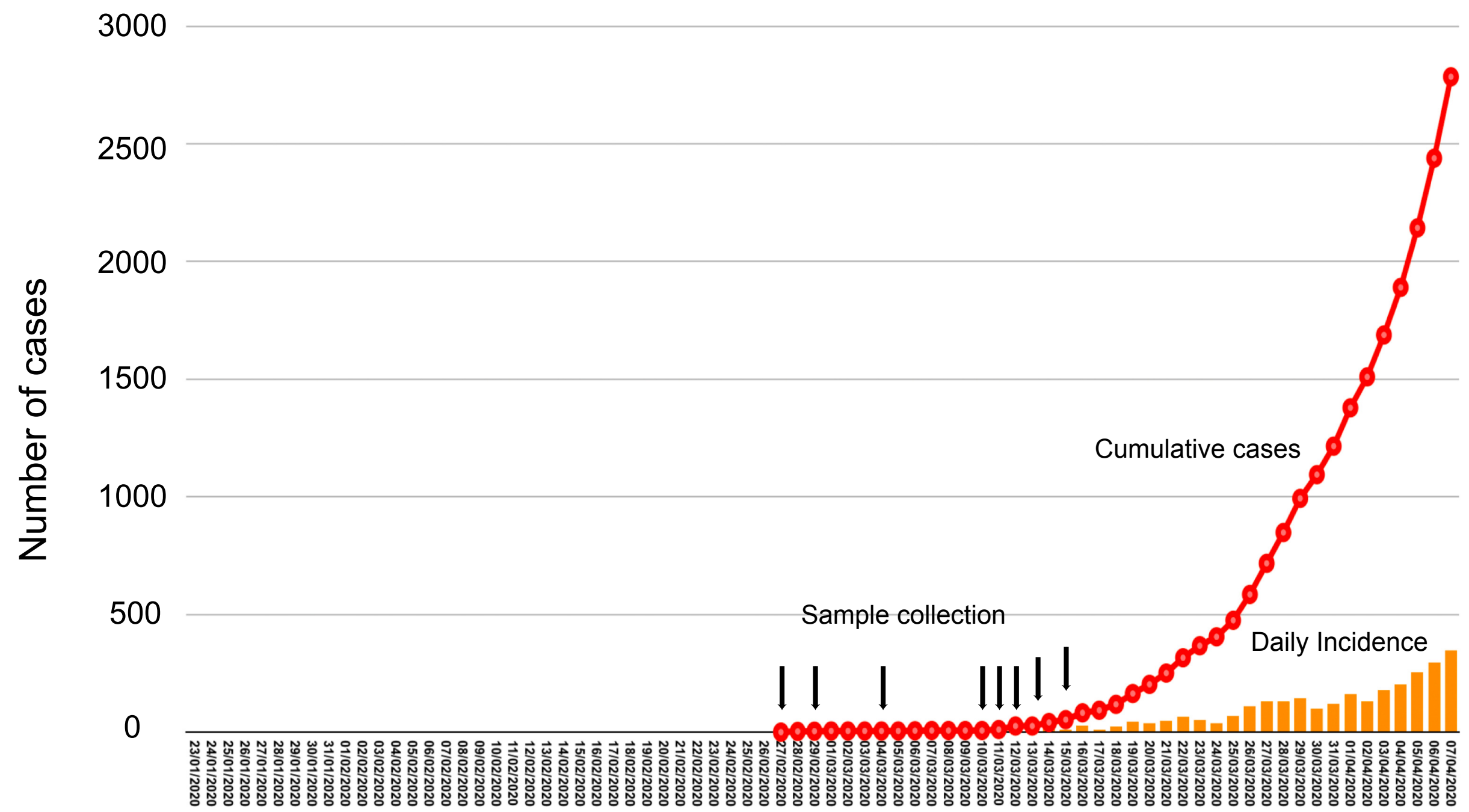

Date of collection 
Figure 2

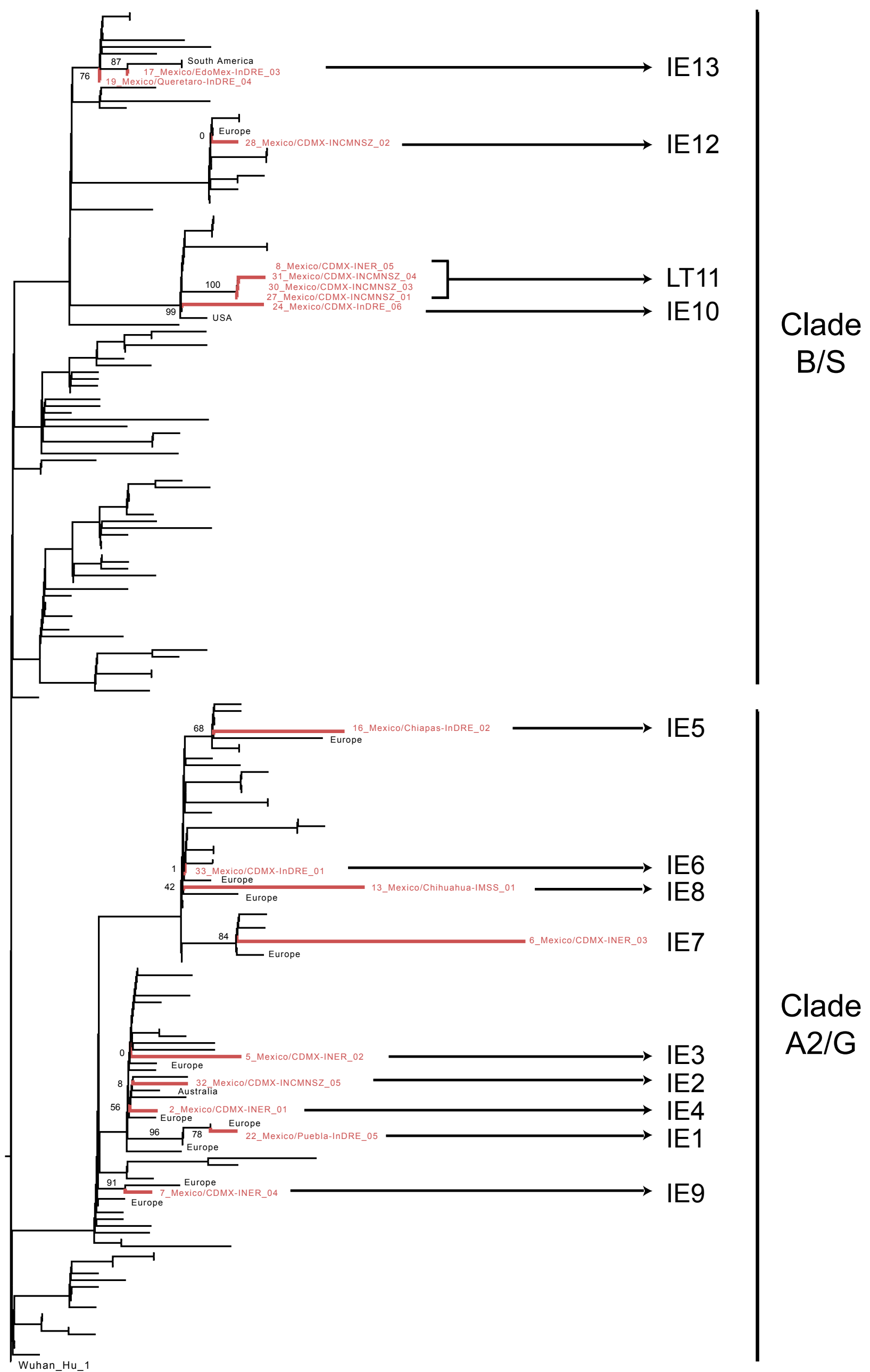


Table 1. List of viral genomes derived from Mexican samples.

\begin{tabular}{|c|c|c|c|c|c|c|c|c|c|}
\hline Sample ID & Virus name & $\begin{array}{l}\text { Accession } \\
\text { GISAID ID }\end{array}$ & $\begin{array}{c}\text { Location } \\
\text { Country_City }\end{array}$ & Age & Sex M/F & EH Place & $\begin{array}{c}\text { Collection } \\
\text { date }\end{array}$ & $\begin{array}{c}\text { Arrival to } \\
\text { Mexico }\end{array}$ & $\begin{array}{c}\text { Port of } \\
\text { Entry }\end{array}$ \\
\hline 2 & Mexico/CDMX-INER 01 & EPI ISL 424345 & Mexico CDMX & 42 & $\bar{M}$ & EH Spain & $12 / 03 / 20$ & $12 / 03 / 20$ & Mexico City \\
\hline 5 & Mexico/CDMX-INER_02 & EPI_ISL_424348 & Mexico_CDMX & 55 & $\mathrm{~F}$ & EH_Spain_France & $13 / 03 / 20$ & $12 / 03 / 20$ & Mexico City \\
\hline 6 & Mexico/CDMX-INER 03 & EPI ISL 424625 & Mexico CDMX & 29 & M & EH Spain & $13 / 03 / 20$ & $11 / 03 / 20$ & Mexico City \\
\hline 7 & Mexico/CDMX-INER_04 & EPI_ISL_424626 & Mexico_CDMX & 25 & $\mathrm{~F}$ & EH_Egypt_Barcelona_Spain & $15 / 03 / 20$ & $15 / 03 / 20$ & Mexico City \\
\hline 8 & Mexico/CDMX-INER_05 & EPI_ISL_424627 & Mexico_CDMX & 38 & M & $\bar{E} \mathrm{H} \_$None & $15 / 03 / 20$ & N/A & N/A \\
\hline 13 & Mexico/Chihuahua-IMSSS_01 & EPI_ISL_424731 & Mexico_Chihuahua & 21 & M & EH_UK_France_USA & $13 / 03 / 20$ & $12 / 03 / 20$ & Mexico City \\
\hline 16 & Mexico/Chiapas-InDRE_02 & EPI_ISL_424666 & Mexico_Chiapas & 18 & $\mathrm{~F}$ & EH_Italy & $29 / 02 / 20$ & $25 / 02 / 20$ & Mexico City \\
\hline 17 & Mexico/EdoMex-InDRE_03 & EPI_ISL_424667 & Mexico_Edomex & 71 & M & $\mathrm{EH}$ _Italy & $04 / 03 / 20$ & $21 / 02 / 20$ & Mexico City \\
\hline 19 & Mexico/Queretaro-InDRE_04 & EPI_ISL_424670 & Mexico_Queretaro & 43 & M & EH_Spain_Holand_USA & $10 / 03 / 20$ & $06 / 03 / 20$ & Mexico City \\
\hline 22 & Mexico/Puebla-InDRE_05 & EPI_ISL_424672 & Mexico_Puebla & 31 & $M$ & $\bar{E} H \_S p a \overline{i n}$ France & $11 / 03 / 20$ & $09 / 03 / 20$ & Mexico City \\
\hline 24 & Mexico/CDMX-InDRE 06 & $\mathrm{EPI}^{-} \mathrm{ISL}^{-} 424673$ & Mexico ${ }^{-}$CDMX & 63 & $\mathrm{~F}$ & $\mathrm{EH}^{-}$Denvē USA & $12 / 03 / 20$ & $06 / 03 / 20$ & Mexico City \\
\hline 27 & Mexico/CDMX-INCMNSZ__01 & EPI_ISL_426361 & México_CDMX & 70 & $\mathrm{~F}$ & ĒH_Vail_ŪSA & $12 / 03 / 20$ & $08 / 03 / 20$ & Mexico City \\
\hline 28 & Mexico/CDMX-INCMNSZ_02 & EPI_ISL_426362 & México_CDMX & 70 & M & EH_Vail_USA & $12 / 03 / 20$ & $08 / 03 / 20$ & Mexico City \\
\hline 30 & Mexico/CDMX-INCMNSZ_03 & EPI_ISL_426363 & México_CDMX & 59 & M & EH_Madrid_Spain & $08 / 03 / 20$ & $12 / 03 / 20$ & Mexico City \\
\hline 31 & Mexico/CDMX-INCMNSZ_04 & EPI_ISL_426364 & México_CDMX & 71 & $\mathrm{M}$ & EH_Vail_USA_Germany & $10 / 03 / 20$ & $08 / 03 / 20$ & Mexico City \\
\hline 32 & Mexico/CDMX-INCMNSZ_05 & EPI_ISL_426365 & México_CDMX & 32 & M & $\overline{\mathrm{EH}}$ _None & $12 / 03 / 20$ & $\mathrm{~N} / \mathrm{A}$ & $\mathrm{N} / \mathrm{A}$ \\
\hline 33 & Mexico/CDMX-InDRE_01 & EPI_ISL_412972 & México_CDMX & 35 & $\mathrm{M}$ & EH_Italy & $27 / 02 / 20$ & N/A & Mexico City \\
\hline
\end{tabular}


Table 2. Nucleotide and amino acid changes in the Mexican samples compared to the reference strain Wuhan-Hu-1.

\begin{tabular}{|c|c|c|c|c|c|}
\hline & \multicolumn{5}{|c|}{ nt change/position in WG nt aln ${ }^{1}$ vs MN908947 } \\
\hline & 241 & 14408 & 18060 & 23403 & 28144 \\
\hline MN908947 & C & C & C & A & $\mathrm{U}$ \\
\hline 7 Mexico/CDMX-INER 04 & $\bar{U}$ & $\bar{U}$ & & $\mathrm{G}$ & \\
\hline $\begin{array}{r}22 \text { Mexico/Puebla-InDRE } 05 \\
2 \text { Mexico/CDMX-INER } 01\end{array}$ & U & u & & G & . \\
\hline $\begin{array}{l}2 \text { Mexico/CDMX-INER } 01 \\
32 \text { Mexico/CDMX-INCMNSZ } 05\end{array}$ & u & $\mathrm{u}$ & . & G & : \\
\hline 5 Mexico/CDMX-INER 02 & U & u & & G & \\
\hline 6 Mexico/CDMX-INER 03 & u & u & & $\mathrm{N}$ & \\
\hline 13 Mexico/Chihuahua-IMSS 01 & U & U & & G & \\
\hline 33 Mexico/CDMX-InDRE 01 & & u & & G & \\
\hline 16 Mexico/Chiapas-InDRE 02 & u & $\mathrm{N}$ & & $\mathrm{N}$ & $\mathrm{N}$ \\
\hline 27 Mexico/CDMX-INCMNSZ_ 01 & & & U & & c \\
\hline $30-$ Mexico/CDMX-INCMNSZ̄ 03 & & & u & & \\
\hline & & & U & . & c \\
\hline $8 \mathrm{Mexico/CDMX-INER} 05$ & & & u & & C \\
\hline & & & U & . & C \\
\hline 28 Mexico/CDMX-INCMNSZ 02 & & & & & C \\
\hline $\begin{array}{l}19 \text { Mexico/Queretaro-InDRE } 04 \\
17 \text { Mexico/EdoMex-InDRE } 03\end{array}$ & & & & & ${ }_{c}^{C}$ \\
\hline eage defining mutations ${ }^{\mathrm{s}}$ & $\overline{A 2 / G}$ & $\overline{A 2 a}$ & B1 & $\overline{\mathrm{A} 2 / \mathrm{G}}$ & B/S \\
\hline
\end{tabular}

\begin{tabular}{|c|c|c|c|c|c|c|c|c|c|c|c|c|c|}
\hline \multirow{3}{*}{\begin{tabular}{c|} 
Genome Region \\
\end{tabular}} & \multicolumn{13}{|c|}{ aa change/position in WG ORF concatenated aln ${ }^{1}$ vs MN908947 } \\
\hline & \multicolumn{4}{|c|}{ Orf1a (1-4405) } & \multicolumn{3}{|c|}{ Orf1b (4406-7000) } & \multicolumn{2}{|c|}{$S(7001-8282)$} & \multirow{2}{*}{\begin{tabular}{|c|} 
Orf3 (8505-8779) \\
8700 \\
\end{tabular}} & \multirow{2}{*}{\begin{tabular}{|c|} 
Orf7a (8780-8900) \\
8817
\end{tabular}} & \multirow{2}{*}{\begin{tabular}{|c|} 
Orf8 (8901-9021) \\
8984
\end{tabular}} & \multirow{2}{*}{\begin{tabular}{|c|}
$\mathrm{N}(9022-9440)$ \\
9225 \\
\end{tabular}} \\
\hline & 224 & 892 & 3071 & 3606 & 4619 & 5696 & 5732 & 7058 & 7623 & & & & \\
\hline MN908947 & $\mathrm{T}$ & $\mathrm{P}$ & $\mathrm{F}$ & $\mathrm{L}$ & $\mathrm{P}$ & $\mathrm{s}$ & $\mathrm{P}$ & $\mathrm{H}$ & $\mathrm{D}$ & G & G & $L$ & G \\
\hline 7_Mexico/CDMX-INER_04 & . & $\mathrm{L}$ & 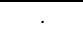 & . & $\mathrm{L}$ & 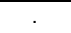 & 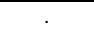 & . & $\bar{G}$ & . & & . & . \\
\hline 22_Mexico/Puebla-InDRE_05 & . & . & $\cdot$ & & $\mathrm{L}$ & $\mathrm{L}$ & 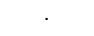 & & G & 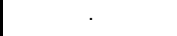 & $\mathrm{v}$ & . & 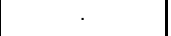 \\
\hline 2_Mexico/CDMX-INER_01 & . & . & . & & $\mathrm{L}$ & . & . & . & G & . & . & . & \\
\hline 32_Mexico/CDMX-INCMNSZ_05 & . & . & . & & $\mathrm{L}$ & . & . & . & G & . & . & . & . \\
\hline$\overline{5}$ _Mexico/CDMX-INER_02 & . & & . & & $\mathrm{L}$ & . & . & & G & . & & . & \\
\hline 6_Mexico/CDMX-INER_03 & . & $\mathrm{x}$ & & & $\mathrm{L}$ & . & . & . & $\mathrm{x}$ & . & $\mathrm{x}$ & . & $\mathrm{x}$ \\
\hline 13_Mexico/Chihuahua-IMSS_01 & . & . & $\mathrm{x}$ & & $\mathrm{L}$ & . & . & & G & . & $\mathrm{x}$ & . & $\mathrm{R}$ \\
\hline$\overline{33} \_$Mexico/CDMX-InDRE_ 01 & & & & . & L & & & . & G & 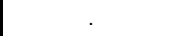 & & & $\mathrm{R}$ \\
\hline 16_Mexico/Chiapas-InDRE_02 & . & 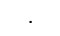 & 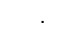 & & $\mathrm{x}$ & . & & & $\mathrm{x}$ & . & $\mathrm{x}$ & $\mathrm{x}$ & $\mathrm{R}$ \\
\hline 27_Mexico/CDMX-INCMNSZ_01 & . & . & . & & . & . & $\mathrm{L}$ & $\mathrm{Y}$ & . & . & . & $\mathrm{s}$ & \\
\hline 30_Mexico/CDMX-INCMNSZ_03 & r. & . & . & & 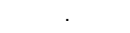 & . & $\mathrm{L}$ & $\mathrm{Y}$ & . & . & . & $\mathrm{s}$ & . \\
\hline 31_Mexico/CDMX-INCMNSZ_04 & & & & 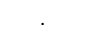 & . & & L & $\mathrm{Y}$ & . & . & . & $\mathrm{s}$ & . \\
\hline 8_Mexico/CDMX-INER_05 & & . & . & & . & 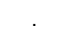 & $\mathrm{L}$ & $\mathrm{Y}$ & . & . & . & $\mathrm{s}$ & 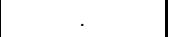 \\
\hline 24_Mexico/CDMX-InDRE_06 & I & . & & $\mathrm{F}$ & . & . & L & . & . & & 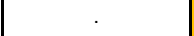 & $\mathrm{s}$ & \\
\hline 28_M̄exico/CDMX-INCMNSZ̄_02 & . & . & $\mathrm{Y}$ & & . & r & & & . & $\mathrm{v}$ & . & $\mathrm{s}$ & . \\
\hline 19_Mexico/Queretaro-InDRE_04 & 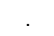 & 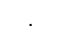 & . & & 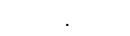 & . & 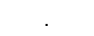 & . & . & & . & $\mathrm{s}$ & \\
\hline 17 Mexico/EdoMex-InDRE 03 & & & & & & & & & & & & $\mathrm{~s}$ & \\
\hline 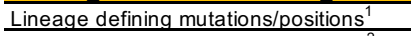 & & & & & A2a (P314L) & & & $\mathrm{H} 49 \mathrm{Y}$ & A2/G (D614G) & & & B/S (L84S) & \\
\hline Non-conservative amino-acid change ${ }^{2}$ & & & & & & & & & & & & & \\
\hline Changes observed $^{2}$ & \# & & $\S$ & WS & WS & $t$ & WS & & WS & $\S$ & $\ddagger$ & WS & WS \\
\hline Frequency ${ }^{3}$ & T99 & P99 & F98 Y2 & L83 F15 & L58 P42 & S99 & P87 L12 & H99 & Y58 D41 & G98 V2 & G99 & L82 S17 & G83 R16 \\
\hline Evidence for $p$ & & & & & & & & & & & & & \\
\hline
\end{tabular}

${ }^{1}$ General lineage defining mutations are shown in blue and orange, as defined in https://nextstrain.org/ncov/global and in GISAID preliminary analysis summary update 2020-04-07 1500UTC. No changes observed in M CDS (8283-8504).

Non-conservative amino-acid changes shown in red

Texican sequences are shown in black.

Widespread mutations are indicated under WS. Unique changes to the Mexican sequences representing singletons are excluded (extended info avilable in SI Table 5).

"Shared with USA/WA_UW153/2020|EPI_ISL_416691|2020_03_13

* Shared with England/SHEF_C0707/2020|EPI_ISL_420270|2020_03_22

$\S$ Shared with 45 sequences, mostly from Australia and Spain.

† Shared with 5 sequences from Australia, Portugal, Spain and USA.

${ }^{\$}$ Shared with USA/WA_UW304/2020|EPI_ISL_418872|2020_03_23

${ }^{3}$ Frequency of a given aa in percentage observed in the aln. Only changes present in $>1 \%$ of the sequences in the aln are shown.

${ }^{4}$ Sites with a dN/dS >1 are shown in green, as compared to the list of sites determined under diverse dN/dS models described in http://covid19.datamonkey.org/2020/04/01/covid19-analysis/ 
Supplementary Table 1. Sequencing results.

\begin{tabular}{cccccccc}
\hline $\begin{array}{c}\text { Sample } \\
\text { ID }\end{array}$ & Virus_name & $\begin{array}{c}\text { Sample } \\
\text { Type }\end{array}$ & $\begin{array}{c}\text { Raw valid } \\
\text { reads }\end{array}$ & $\begin{array}{c}\text { On-target } \\
\text { reads ( \%) }\end{array}$ & $\begin{array}{c}\text { Genome } \\
\text { coverage }\end{array}$ & Depth & Mean Depth* \\
\hline 2 & Mexico/CDMX-INER_01 & NPS & $13,949,246$ & $12,406(0.09)$ & 99.17 & 30 & 30 \\
5 & Mexico/CDMX-INER_02 & NPS & $26,958,293$ & $28,208(0.10)$ & 98.56 & 69 & 70 \\
6 & Mexico/CDMX-INER_03 & NPS & $11,143,321$ & $3,970(0.04)$ & 84.25 & 9 & 11 \\
7 & Mexico/CDMX-INER_04 & NPS & $10,372,811$ & $21,080(0.20)$ & 99.82 & 51 & 51 \\
8 & Mexico/CDMX-INER_05 & NPS & $2,274,643$ & $11,648(0.51)$ & 99.73 & 28 & 28 \\
13 & Mexico/Chihuahua-IMSS_01 & NPS & $12,785,238$ & $7,331(0.06)$ & 90.98 & 18 & 20 \\
16 & Mexico/Chiapas-InDRE_02 & OPS & $4,322,780$ & $1,739(0.04)$ & 83.06 & 4 & 8 \\
17 & Mexico/EdoMex-InDRE_03 & NAS & $8,173,886$ & $85,170(1.04)$ & 99.90 & 200 & 200 \\
19 & Mexico/Queretaro-InDRE_04 & OPS & $9,394,551$ & $60,545(0.64)$ & 99.91 & 145 & 145 \\
22 & Mexico/Puebla-InDRE_05 & OPS & $5,030,534$ & $39,210(0.78)$ & 99.90 & 93 & 93 \\
24 & Mexico/CDMX-InDRE_06 & OPS & $3,890,600$ & $109,000(2.81)$ & 99.92 & 256 & 256 \\
27 & Mexico/CDMX-INCMNSZ_01 & NPS & $14,770,367$ & $79,364(0.54)$ & 99.88 & 164 & 164 \\
28 & Mexico/CDMX-INCMNSZ_02 & NPS & $2,898,415$ & $21,985(0.76)$ & 99.90 & 53 & 53 \\
30 & Mexico/CDMX-INCMNSZ_03 & NPS & $3,956,195$ & $23,550(0.60)$ & 99.91 & 57 & 57 \\
31 & Mexico/CDMX-INCMNSZ_04 & NPS & $4,581,562$ & $97,388(2.13)$ & 99.92 & 228 & 19 \\
32 & Mexico/CDMX-INCMNSZ_05 & NPS & $2,648,330$ & $7,813(0.30)$ & 99.34 & 228 \\
33 & Mexico/CDMX-InDRE_01 & NPS & $2,662,304$ & $1,000,109(37.57)$ & 100.00 & 3983 & 19 \\
\hline
\end{tabular}

\#Abbreviation: NPS=nasopharyngeal swab, OP= oropharyngeal swab, NAS=nasopharyngeal aspirate

* In relationship to the ratio of unambiguous bases to the reference sequence length (using at least 5 unique reads and $90 \%$ agreement during base calling) 


\begin{tabular}{|c|c|c|c|c|}
\hline reduced $-\mathbf{W G}^{1}$ & large-scale WG ${ }^{1}$ & large-scale Orf1 $\mathrm{a}^{1}$ & large-scale Orf1 $b^{1}$ & large-scale $\mathrm{S}^{1}$ \\
\hline 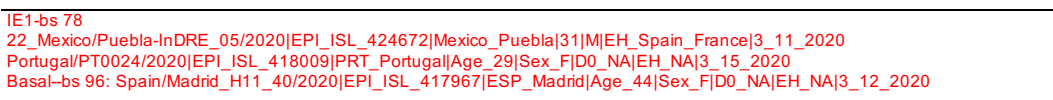 & $\begin{array}{l}\text { IE1-bs } 6 \\
\text { YES }\end{array}$ & $\begin{array}{l}\text { IE1-bs } 0 \\
\text { NO }\end{array}$ & $\begin{array}{l}\text { IE1-bs } 0 \\
\text { YES }\end{array}$ & $\begin{array}{c}\text { IE1-bS } 0 \\
\text { NO-POLYTOMY }\end{array}$ \\
\hline 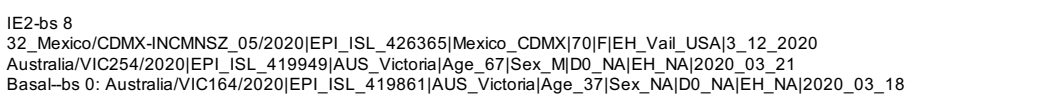 & $\begin{array}{l}\text { IE2-bs } 27 \\
\text { YES }\end{array}$ & $\begin{array}{l}\text { IE2-bs } 0 \\
\text { NO }\end{array}$ & $\begin{array}{l}\text { IE2-bs } 0 \\
\text { NO }\end{array}$ & $\begin{array}{l}\text { IE2-bs } 0 \\
\text { NO }\end{array}$ \\
\hline 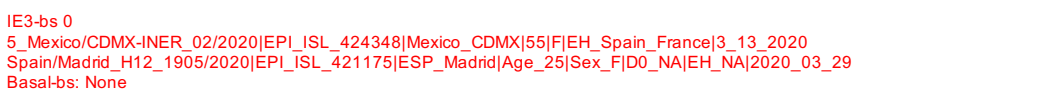 & $\begin{array}{l}\text { IE3-bs } 0 \\
\text { YES }\end{array}$ & $\begin{array}{l}\text { IE3-bs } 0 \\
\text { NO }\end{array}$ & $\begin{array}{l}\text { IE3-bs } 0 \\
\text { YES-PLLYTOMY }\end{array}$ & $\begin{array}{l}\text { IE3-bs } 0 \\
\text { NO }\end{array}$ \\
\hline $\begin{array}{l}\text { IE4-bs } 56 \\
\text { 2_Mexico/CDMX-INER_01/2020|EPI_ISL_424345|Mexico_CDMX|142|M|EH_Spain|3_12_2020 } \\
\text { Hungary/2/2020|EPI_ISL_418183|HUN_Baranyal|Age_26|Sex_F|DO_H|EH_NA|2020_03_17 } \\
\text { Basal-bs } 0 \text { : None }\end{array}$ & $\begin{array}{l}\text { IE4-bs } 61 \\
\text { NO }\end{array}$ & $\begin{array}{l}\text { IE4-bs } 0 \\
\text { NO }\end{array}$ & $\begin{array}{c}\text { IE4-bs } 0 \\
\text { NO-POLYTOMY }\end{array}$ & $\begin{array}{l}\text { IE4-bs } 0 \\
\text { NO }\end{array}$ \\
\hline $\begin{array}{l}\text { IE5-bs } 68 \\
\text { Waless/PHWC_25551/2020|EPI_ISL_421004|GBR_Wales|Age_73|Sex_MIDO_NA|EH_NA|2020_03_23 } \\
\text { 16_Mexico/Chiapas-InDRE_02/2020|EPI_ISL_424666|Mexico_Chiapas|18|F|F|EH_taly|2_29_2020_2 } \\
\text { Basal-bs 0: None }\end{array}$ & $\begin{array}{l}\text { IE5-bs } 19 \\
\text { YES }\end{array}$ & $\begin{array}{l}\text { IE5-bs } 0 \\
\text { NO }\end{array}$ & $\begin{array}{l}\text { IE5-bs } 0 \\
\text { NO }\end{array}$ & $\begin{array}{c}\text { IE5-bs } 0 \\
\text { NO-POLYTOMY }\end{array}$ \\
\hline $\begin{array}{l}\text { IE6-bs } 0 \\
\text { 33_Mexico/CDMX-InDRE_01/2020|EPI_ISL_4 412972|Mexico_CDMX|35|M|EH_Italy|2_27_2020 } \\
\text { Basal-bs 1:England/20122119502/2020|EPI_ISL_418681|GBR_England|Age_31|Sex_F|DO_NA|EH_NA|2020_03_17 }\end{array}$ & $\begin{array}{l}\text { IE6-bs } 1 \\
\text { NO }\end{array}$ & $\begin{array}{c}\text { IE6-bs } 0 \\
\text { NO-POLYTOMY }\end{array}$ & $\begin{array}{c}\text { IE6-bs } 0 \\
\text { NO-POLYTOMY }\end{array}$ & $\begin{array}{c}\text { IE6-bs } 0 \\
\text { NO-POLYTOMY }\end{array}$ \\
\hline $\begin{array}{l}\text { IE7-bs } 84 \\
\text { 6_Mexico/CDMX-INER_03/2020|EPI_ISL_424625|Mexico_CDMX|29|M|EH_Spain|3-13_2020 } \\
\text { England/20108034006/2020|EPI_ISL_417258|GBR_England|Age_74|SeX_FIDO_NA|EH_NA|2020_03_06 }\end{array}$ & $\begin{array}{l}\text { IE7-bs } 67 \\
\text { YES }\end{array}$ & $\begin{array}{l}\text { IE7-bs } 0 \\
\text { YES }\end{array}$ & $\begin{array}{l}\text { IE7-bs } 27 \\
\text { YES }\end{array}$ & $\begin{array}{l}\text { IE7-bs } 0 \\
\text { NO }\end{array}$ \\
\hline $\begin{array}{l}\text { IE8-bs 42 } \\
\text { Italy|TE4959/2020|EPI_ISL_418259||TA_Abruzzo|Age_76|Sex_MIDO_NA|EH_NA|2020_03_14 } \\
\text { 13_Maxico/Chinuahua-IMSS_01/2020|EPI_ISL_424731|Mexico_Chihuahua|21||M|EH_UKK_France_USA|3_13_2020 } \\
\text { Basal-bs 0: None }\end{array}$ & $\begin{array}{l}\text { IE8-bs } 18 \\
\text { YES }\end{array}$ & $\begin{array}{l}\text { IE8-bs } 0 \\
\text { NO }\end{array}$ & $\begin{array}{c}\text { IE8-bs } 0 \\
\text { NO-POLYTOMY }\end{array}$ & $\begin{array}{c}\text { IE8-bs } 0 \\
\text { NO-POLYTOMY }\end{array}$ \\
\hline $\begin{array}{l}\text { IE9-bs } 91 \\
\text { 7_Mexico//CDMX-INER_04/2020|EPI_ISL_424626|Mexico_CDMX|25|F|EH_Egypt_Barcelona_Spain|3_15_2020 } \\
\text { England/SHEF_C0707/2020|EPI_ISL_420270|GBR_England|Age_73|SeX_FIDO_NA|EH_NA|2020_03_22 } \\
\text { Basal-bs: None }\end{array}$ & $\begin{array}{l}\text { IE9-bs } 79 \\
\text { YES }\end{array}$ & $\begin{array}{l}\text { IE9-bs } 0 \\
\text { YES }\end{array}$ & $\begin{array}{c}\text { IE9-bs } 0 \\
\text { NO-POLYTOMY }\end{array}$ & $\begin{array}{l}\text { IE9-bs } 0 \\
\text { NO }\end{array}$ \\
\hline $\begin{array}{l}\text { IE10-bs } 99 \\
\text { Australia/VIC110/2020|EPI_ISL_419716|AUS_Victoria|Age_62|Sex_FIDO_NA|EH_NA|2020_03_18 } \\
\text { 24_Mexico/CDMX-InDRE_06/2020|EPI_ISL_424673|Mexico_CDMX|63|FIEH_Denver_USA|3_12_2020 }\end{array}$ & $\begin{array}{l}\text { IE10-bs } 0 \\
\text { NO }\end{array}$ & $\begin{array}{c}\text { IE10-bs } 2 \\
\text { YES }\end{array}$ & $\begin{array}{c}\text { IE10-bs 0 } \\
\text { YES-POLYTOMY }\end{array}$ & $\begin{array}{l}\text { IE10-bs 0 } \\
\text { YES-POLYTOMY }\end{array}$ \\
\hline 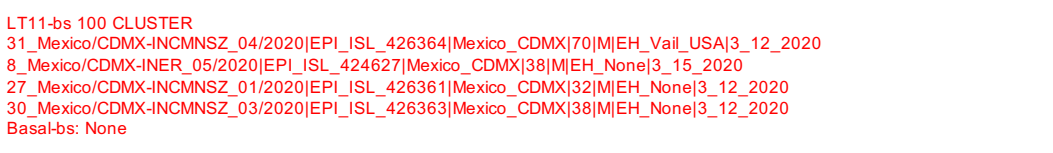 & $\begin{array}{l}\text { IE11-bs } 98 \text { CLUSTER } \\
\text { YES }\end{array}$ & $\begin{array}{c}\text { C11.1-bs } 0 \\
\text { BROKEN CLUSTER }\end{array}$ & $\begin{array}{c}\text { C11.1-bs } 0 \\
\text { BROKEN CLUSTER }\end{array}$ & $\begin{array}{c}\text { IE11-bs } 89 \text { CLUSTER } \\
\text { YES }\end{array}$ \\
\hline 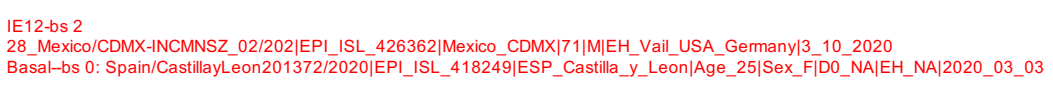 & $\begin{array}{l}\text { IE12-bs } 0 \\
\text { YES }\end{array}$ & $\begin{array}{l}\text { IE12-bs } 0 \\
\text { YES }\end{array}$ & $\begin{array}{c}\text { IE12-bs } 0 \\
\text { YES-POLYTOMY }\end{array}$ & $\begin{array}{l}\text { IE12-bs } 0 \\
\text { NO }\end{array}$ \\
\hline 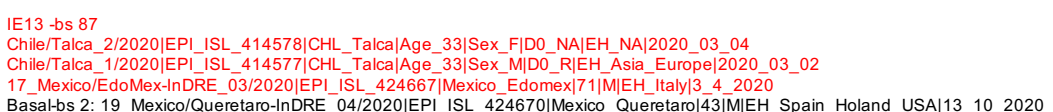 & $\begin{array}{l}\text { IE13-bs } 0 \\
\text { YES }\end{array}$ & $\begin{array}{l}\text { IE13-bs } 0 \\
\text { YES }\end{array}$ & $\begin{array}{l}\text { IE13-bs } 33 \\
\text { YES }\end{array}$ & $\begin{array}{l}\text { IE13-bs } 0 \\
\text { NO }\end{array}$ \\
\hline
\end{tabular}

${ }^{1}$ Consistency for clustering patterns observed within $>2$ trees and/or bootstrap support values $>50$ are indicated in red. YES/NO indicates conserved clustering patterms. 


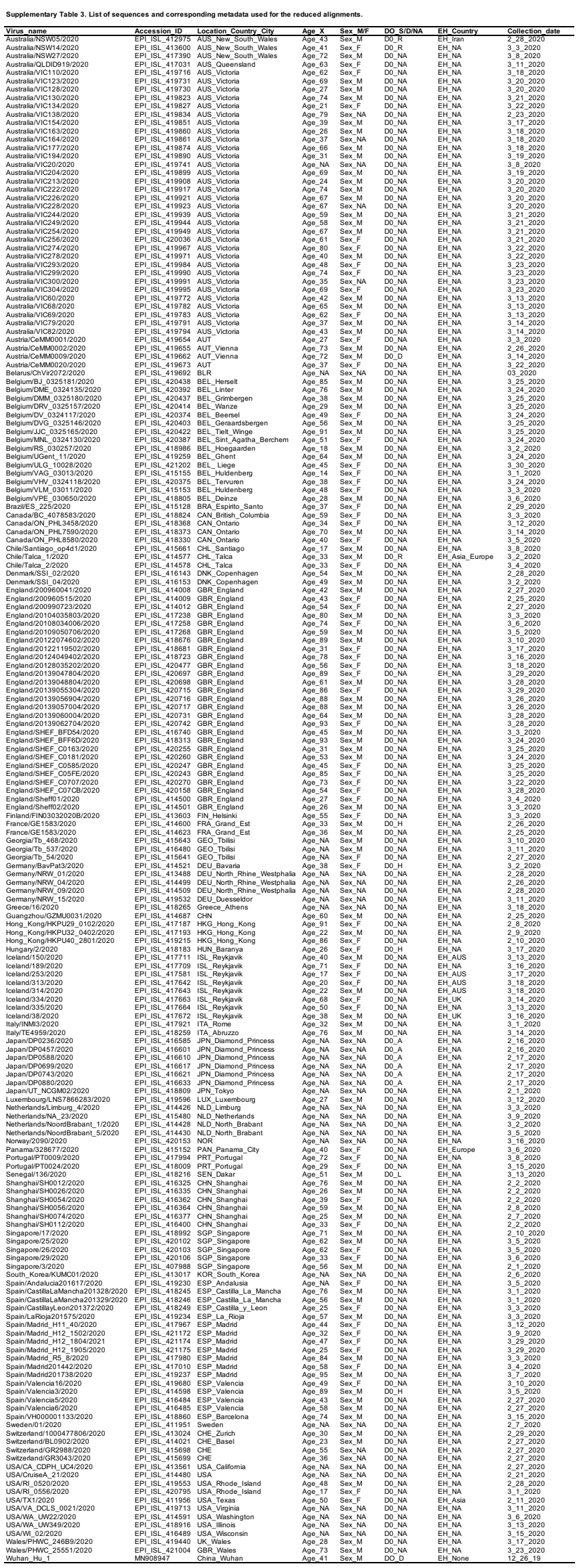




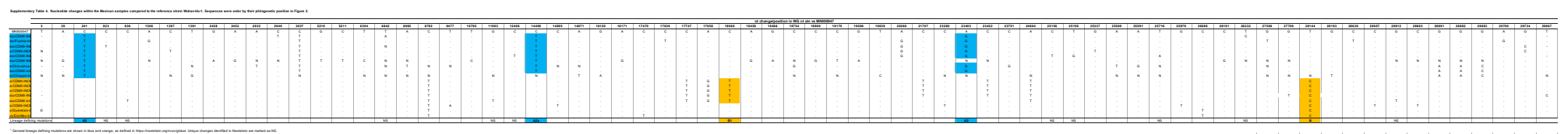

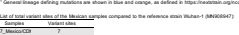

急

출 


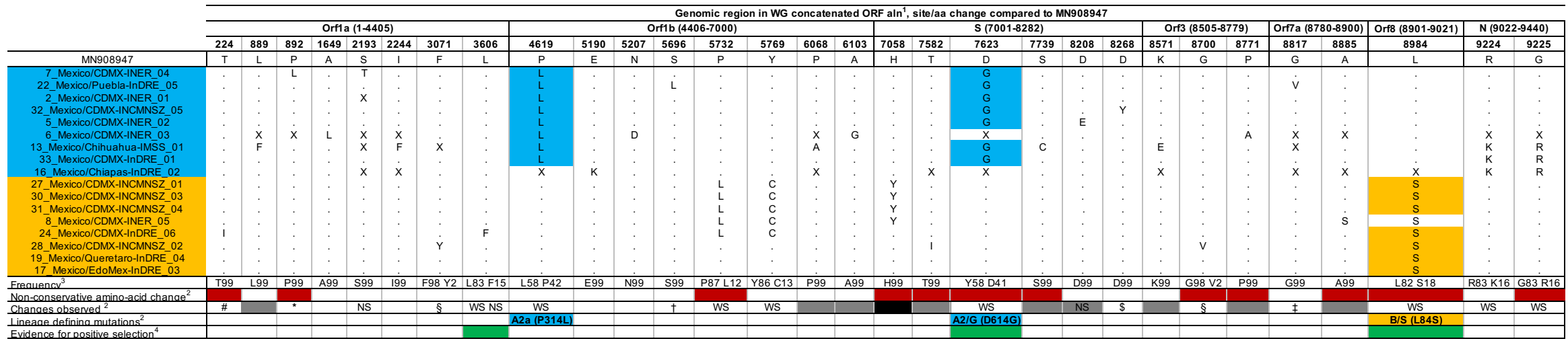

'General lineage defining mutations are shown in blue and orange, as defined in https://nextstrain.org/ncov/global and in GISAID preliminary analysis summary update 2020-04-08 1500UTC. No changes observed in M CDS (8283-8504).

Non-conservative amino-acid changes shown in red
Cluster defining homoplassic mutation of the Mexican

Widespread mutations are indicated under WS. Mutations also in Nexstrain are marked as NS. Unique changes to he Mexican sed

* Shared with England/SHEF_C0707/2020||EPI_ISL_420270|2020_03_22

\$ S Shared with 45 sequencess, mostly from $\mathrm{S}$ Spain.

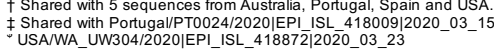

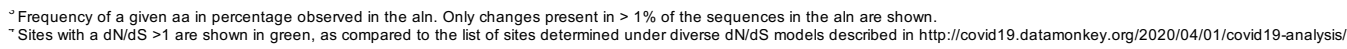

